Ann. Biol. anim. Bioch. Biophys., I974, 14 (4-A), 679-687.

\title{
VARIATIONS QUALITATIVES ET QUANTITATIVES DE LA FAUNE DU RUMEN DU MOUTON EN FONCTION DE LA NATURE DU RÉGIME
}

\author{
Annie BONHOMME et Michelle DURAND* \\ avec la collaboration technique de Ph. Beaumatin et F. Le Deschault de Montredon \\ Laboratoire de Zoologie, Faculté des Sciences, \\ 51062 Reims Cedex \\ * Station de Recherches de Nutrition, \\ Centre national de Recherches zootechniques, I. N. R. A., \\ 78350 Jouy en Josas
}

\section{RÉSUMÉ}

Au cours d'études in vivo, nous avons observé les variations qualitatives et quantitatives de la population de Protozoaires en fonction de différentes rations distribuées en deux repas quotidiens. Ce sont les régimes à base d'orge-urée qui favorisent le développement le plus important des Ciliés essentiellement du genre Entodinium. Les régimes composés de foin de graminées favorisent une population plus variée dans les genres dont en majorité des grandes espèces, mais quantitativement dix fois moindre environ.

Des mesures de pH et de concentration d'azote ammoniacal du rumen ont été réalisées pour étudier les relations entre certaines conditions chimiques du rumen et le développement de la microfaune.

\section{I. - INTRODUC'TION}

Lorsque l'on étudie la microfaune du rumen et ses relations avec l'hôte, il faut tenir compte de plusieurs facteurs qui font varier cette micropopulation.

Ce sont les facteurs géographiques, saisonniers et alimentaires. Ceux-ci comprennent : la composition chimique de la ration, le nombre des repas et les intervalles de temps entre les repas. D'autres facteurs interviennent également, ils dépendent de l'hôte lui-même : le facteur individuel, (habitudes nutritives du mouton, taux d'ingestion de la nourriture), l'âge et la santé de l'animal. Et si l'on considère différentes espèces de Ruminants voire même plus largement des Herbivores, cette faune présente une certaine spécificité pour l'hôte (NAGA et coll., I969). D'autre part, des modifications importantes de la flore bactérienne peuvent être un des principaux facteurs des fluctuations quantitatives et qualitatives des Ciliés. I.es Bac- 
téries sont une source nutritive indispensable aux Ciliés; in vitro, en leur absence, les Protozoaires disparaissent (Bоnноммe, I973). Dans le rumen un équilibre s'établit entre ces deux micropopulations qui sont en compétition pour la nourriture. Enfin la composition qualitative de la microfaune dépend également des antagonismes existant entre les différentes espèces de Ciliés la composant (EADIE, I967). Au cours de nos précédents travaux (Bonнomme, I973) la faune du rumen que nous utilisions provenait de moutons fraîchement tués aux abattoirs. Nous avions remarqué, d'après les particules alimentaires résiduelles, combien cette faune variait quantitativement et qualitativement en fonction de la ration.

Aussi avons-nous abordé l'étude de l'influence de l'alimentation sur la composition de la micropopulation des Protozoaires du rumen. Nous avons dans le présent travail comparé un régime comportant du fourrage (foin de graminées) distribué soit seul, soit avec du tourteau de soja, et un régime comportant une céréale (orge) complémentée par de l'urée.

\section{II. - PROCESSUS EXPÉRIMENTAL}

Cette étude est réalisée sur 3 lots de 3 moutons adultes, munis d'une fistule de rumen, recevant les trois différents régimes après une période d'adaptation de trois semaines. Les rations journalières sont distribuées en deux repas dont la durée est d'une heure. La composition des régimes alimentaires est inscrite dans le tableau $\mathbf{~}$.

TABLEAU I

Composition des régimes alimentaires

\begin{tabular}{|c|c|c|c|c|c|c|}
\hline \multicolumn{3}{|c|}{ Régime I } & \multicolumn{2}{|l|}{ Régime II } & \multicolumn{2}{|c|}{ Régime III } \\
\hline \multicolumn{3}{|c|}{ Moutons $\mathrm{n}^{\mathrm{os}} 68,32,70$} & \multicolumn{2}{|c|}{ Moutons nos $142,29,53$} & \multicolumn{2}{|c|}{ Moutons nos $146,73,59$} \\
\hline Matin & Foin de graminées & $500 \mathrm{~g}$ & $\begin{array}{l}\text { Tourteau de soja } \\
\text { Foin de graminées }\end{array}$ & $\begin{array}{l}100 \mathrm{~g} \\
300 \mathrm{~g}\end{array}$ & $\begin{array}{l}\text { Orge } \\
\text { Urée } \\
\text { Paille }\end{array}$ & $\begin{array}{r}350 \mathrm{~g} \\
19 \mathrm{~g}\end{array}$ \\
\hline Soir & Foin de graminées & $500 \mathrm{~g}$ & Paille à volonté & & $\begin{array}{l}\text { Orge } \\
\text { Paille à } \\
\text { volonté }\end{array}$ & $300 \mathrm{~g}$ \\
\hline
\end{tabular}

Les prélèvements de contenus de rumen sont effectués au cours de quatre jours consécutifs, juste avant le repas ( $\mathrm{To}$ ) et $2 \mathrm{~h} 30$ après le repas ( $\mathrm{T} 2 \mathrm{~h} 30$ ) du matin ou du soir.

On réalise ensuite la détermination des espèces et le dénombrement dans les échantillons de contenus de rumen de chacun des moutons à l'aide d'une cellule de comptage de Bürker.

Les mesures de $\mathrm{pH}$, des quantités d'eau absorbée ct des concentrations en azote ammoniacal sont également effectuées aux temps $\mathrm{T}_{0}$ et $\mathrm{T} 2 \mathrm{~h} 30$. La concentration d'azote ammoniacal est déterminée par la méthode de Conway. 


\title{
III. - RÉSULTATS
}

\section{I. - Etude qualitative de la microfaune}

La variation des genres et espèces des Ciliés en fonction du régime alimentaire est inscrite dans le tableau 2.

\author{
TABLEAU 2
}

Variations qualitatives de la faune en fonction du régime

\begin{tabular}{|c|c|c|c|c|c|c|c|c|c|}
\hline \multirow[b]{3}{*}{ Moutons $\mathrm{n}^{\circ}$} & \multicolumn{3}{|c|}{ Régime I } & \multicolumn{3}{|c|}{ Régime II } & \multicolumn{3}{|c|}{ Régime III } \\
\hline & \multicolumn{3}{|c|}{ Foin de graminées } & \multicolumn{3}{|c|}{$\begin{array}{l}\text { Foin de graminées } \\
\text { Tourteau de soja }\end{array}$} & \multicolumn{3}{|c|}{ Orge-urée } \\
\hline & 70 & 68 & 32 & $1 / 42$ & 53 & 29 & 146 & 73 & 59 \\
\hline Espèces observées & & & & & & & & & \\
\hline $\begin{array}{l}\mathrm{SCl} \text {. Spirotriches } \\
\text { G. Entodinium }\end{array}$ & & & & & & & & & \\
\hline 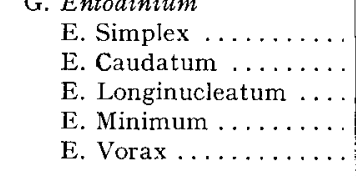 & $\begin{array}{l}+ \\
+\end{array}$ & $\begin{array}{l}+ \\
+\end{array}$ & $\begin{array}{l}+ \\
+\end{array}$ & $\begin{array}{l}+ \\
+ \\
+\end{array}$ & $\begin{array}{l}+ \\
+ \\
+ \\
+ \\
+\end{array}$ & $\begin{array}{l}+ \\
+\end{array}$ & $\begin{array}{l}+ \\
+ \\
+ \\
+\end{array}$ & $\begin{array}{l}+ \\
+ \\
+ \\
+ \\
+\end{array}$ & $\begin{array}{l}+ \\
+\end{array}$ \\
\hline $\begin{array}{l}\text { G. Diplodinium } \\
\text { Diplosplastron affine ... } \\
\text { Polyplastron multivesi- }\end{array}$ & & + & + & $\begin{array}{l}+ \\
+\end{array}$ & $\begin{array}{l}+ \\
+\end{array}$ & + & $t$ & + & . \\
\hline $\begin{array}{c}\text { Anoplodinium, denticu- } \\
\text { latum, anacanthum } \\
\text { Eudiplodinium magii... }\end{array}$ & $\begin{array}{l}t- \\
t-\end{array}$ & & & $T$ & ${ }^{+}$ & & & & \\
\hline $\begin{array}{l}\text { G. Epidinium } \\
\text { E. Ecaudatum }\end{array}$ & + & & & & & & & & \\
\hline $\begin{array}{l}\text { G. Ophryoscolex } \\
\text { O. Tricoronatus .... }\end{array}$ & & & & & & & & & \\
\hline $\begin{array}{l}\text { SCl. Holotriches } \\
\text { Dasytricha } \quad \text { ruminan- } \\
\text { tium } \ldots \ldots \ldots \ldots \ldots\end{array}$ & + & + & + & + & + & + & + & + & + \\
\hline Isotricha prostoma .... & + & + & + & + & + & t- & + & + & + \\
\hline
\end{tabular}

On observe que les grandes espèces du genre Diplodinium sont surtout représentées dans les régimes de fourrage, alors que dans le régime de céréale, les petites espèces du genre Entodinium prédominent. 


\section{2. - Étude quantitative de la microfaune du rumen}

a) Étude quantitative de la population ciliaire globale en fonction du régime.

Les résultats correspondant à la moyenne obtenue sur quatre jours de prélèvement sont les suivants :

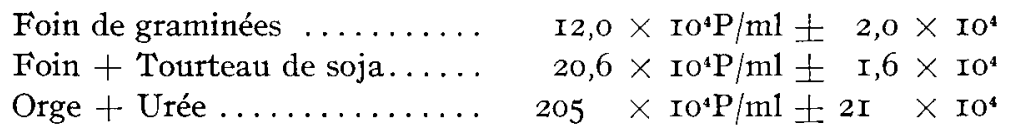

L'on note que la population est la plus dense dans le régime orge-urée, environ dix fois plus que dans les autres régimes à base de fourrage.

b) Étude quantitative de la population ciliaire globale en fonction du temps de prélèvement.

Les résultats sont inscrits dans le tableau 3.

Un mouton n'a pas été utilisé, le 59 du régime III car l'animal est tombé malade au cours de l'expérience. Par ailleurs, le 32 a une population relativement faible.

Il apparaît qu'il est difficile d'établir une relation entre la proportion de Ciliés et le temps de 1'échantillonnage.

TABLEAU 3

Dénombrements de la population ciliaire avant et après le repas du matin et du soir

\begin{tabular}{|c|c|c|c|c|c|}
\hline \multirow{3}{*}{ Régimes } & \multirow{3}{*}{ Moutons $n^{0}$} & \multicolumn{4}{|c|}{ Population $\times 10^{4} \mathrm{P} / \mathrm{mI}$} \\
\hline & & \multicolumn{2}{|c|}{ Matin } & \multicolumn{2}{|c|}{ Soir } \\
\hline & & $T_{0}$ & $\mathrm{~T} 2 \mathrm{~h} 30$ & $\Gamma_{0}$ & $\mathrm{~T}: \mathrm{h} 30$ \\
\hline I & $\begin{array}{l}68 \\
70 \\
32\end{array}$ & $\begin{array}{c}11,3 \\
7 \\
2,5\end{array}$ & $\begin{array}{r}29,9 \\
23,8 \\
2,6\end{array}$ & $\begin{array}{c}15 \\
9,4 \\
1,9\end{array}$ & $\begin{array}{r}18,3 \\
19,0 \\
1,5\end{array}$ \\
\hline II & $\begin{array}{r}29 \\
142 \\
53\end{array}$ & $\begin{array}{l}15,8 \\
22,7 \\
17,8\end{array}$ & $\begin{array}{l}21,4 \\
26,4 \\
35,2\end{array}$ & $\begin{array}{l}10,5 \\
17,7 \\
29,8\end{array}$ & $\begin{array}{l}14,9 \\
22,1 \\
19,6\end{array}$ \\
\hline III & $\begin{array}{r}73 \\
146\end{array}$ & $\begin{array}{l}173 \\
225\end{array}$ & $\begin{array}{l}167 \\
208\end{array}$ & $\begin{array}{l}162 \\
340\end{array}$ & $\begin{array}{l}113,3 \\
2 \cdot 20\end{array}$ \\
\hline
\end{tabular}

On peut noter une légère variation quantitative de la population entre les repas du matin et du soir pour un même régime et un même animal. D'autre part, pour un même régime, on observe une différence quantitative de la population d'un animal à un autre, c'est le cas des moutons du régime III, mais aussi celui du mouton $n^{\circ} 32$ du régime I dont la microfaune est faiblement représentée. 


\section{3. - Infuence des facteurs du milieu}

Nous rapportons ici à titre d'exemple les résultats concernant l'absorption d'eau et la valeur du $\mathrm{pH}$ et des concentrations ammoniacales des contenus de rumen pour un animal par régime, pour lequel la population de Ciliés est la plus abondante (tabl, 4).

La quantité d'eau absorbée entre les temps $T_{0}$ et $T 2 h$ zo varie beaucoup en fonction du régime et de l'heure du repas.

TABLEAU 4

Absorption d'eau et valeurs du pH

et des concentrations en $\mathrm{N}-\mathrm{NH}_{3}$ des contenus de rumen

\begin{tabular}{|c|c|c|c|c|c|c|c|c|c|c|c|}
\hline \multirow{3}{*}{ Régimes } & \multirow{3}{*}{ Moutons } & \multicolumn{2}{|c|}{$\begin{array}{l}\text { Absorption d'eau } \\
\text { entre } \mathrm{T}_{0} \text { et } \mathrm{T} 2 \mathrm{~h} 30 \\
(\mathrm{ml})\end{array}$} & \multicolumn{4}{|c|}{$\mathrm{pH}$} & \multicolumn{4}{|c|}{ Teneur en $\mathrm{N}-\mathrm{NH}_{3} \mathrm{mg} \% \mathrm{ml}$} \\
\hline & & \multirow{2}{*}{ matin } & \multirow{2}{*}{ soir } & \multicolumn{2}{|c|}{ matin } & \multicolumn{2}{|r|}{ soir } & \multicolumn{2}{|c|}{ matin } & \multicolumn{2}{|c|}{ soir } \\
\hline & & & & $\mathrm{T}_{\mathrm{0}}$ & T 2 h 30 & $\mathrm{~T}_{0}$ & $\mathrm{~T} 2 \mathrm{~h} 30$ & $\mathrm{~T}_{0}$ & $\mathrm{~T} 2 \mathrm{~h} 30$ & $\mathrm{~T}_{0}$ & $\mathrm{~T} 2 \mathrm{~h}$ \\
\hline I & 68 & 1057 & 50 & 6,9 & 6,7 & 6,6 & 6,6 & 5,7 & 5,9 & 4,8 & 7,0 \\
\hline II & 142 & 862 & 1060 & 7,0 & 6,8 & 6,9 & 6,9 & 5,7 & 12,7 & 17,5 & 13,7 \\
\hline III & 146 & 390 & 1550 & 6,8 & 6,6 & 6,5 & 6,1 & 9,2 & 28,8 & 23,9 & 8,5 \\
\hline
\end{tabular}

Sur l'ensemble des essais, l'on constate que le $\mathrm{pH}$ est le plus élevé dans le régime foin de graminées (I) et le régime tourteau de soja + foin (II). Le pH est le plus bas dans le régime orge-urée, la fermentation de l'amidon en étant responsable. On observe également que l'azote ammoniacal augmente beaucoup après le repas du matin en ce qui concerne les régimes II et plus encore III, alors qu'il diminue après le repas du soir, où sont absents le tourteau de soja et l'urée. En ce qui concerne le régime I, la valeur est approximativement constante.

\section{IV. - DISCUSSION ET CONCLUSION}

\section{I. - Influence de l'heure des prélèvements et de la consommation d'eau}

La fluctuation diurne du nombre des microorganismes est connue de nombreux auteurs et 1'amplitude et le taux de cette fluctuation varient avec la nature de la ration. WARNER (I962) étudiant les changements diurnes de la micropopulation en fonction des repas, note que le maximum de concentration est atteint à des taux différents pour différents microorganismes. Ainsi, il observe pour Entodinium un temps de division minimum de cinq heures et le taux maximum correspond environ à quatre générations par jour. Parmi les facteurs intervenant pour faire varier la 
population de Ciliés, le $\mathrm{pH}$ jouerait un rôle important. Cependant, nos essais ne sont pas échelonnés sur une période suffisamment longue pour observer des variations diurnes.

En ce qui concerne l'influence de la quantité d'eau absorbée, nous avons pu observer que la proportion des Ciliés dépend du taux de dilution du flux passant du rumen vers l'omasum.

Ainsi, dans les régimes à base de céréales où la population est habituellement très dense en Ciliés du genre Entodinium, lorsque l'animal absorbe entre $\mathrm{T}_{0}$ et $\mathrm{T} 2 \mathrm{~h} 30$ une grande quantité d'eau $(I, 51)$ on observe une baisse de la population ciliaire de 1'ordre de $120 \times \mathrm{IO}^{4} \mathrm{P} / \mathrm{ml}$.

\section{2. - Influence des régimes alimentaires}

Infuence de la nature du régime sur la composition faunale du rumen.

Aspects qualitatif et quantitatif.

Qualitativement.

Dans les régimes foin de graminées, supplémentés ou non en tourteau de soja, on observe pour ainsi dire tous les genres, mais les grandes espèces et plus particulièrement celles du genre Diplodinium sont plus représentées que dans le régime orge-urée où les petites espèces du genre Entodinium prolifèrent. L'espèce $E$. minimum a été strictement observée dans ce régime. Par contre, l'espèce $E$. caudatum qui, selon de nombreux auteurs, est uniquement amylolytique, se rencontre aussi dans le régime foin de graminées.

I1 est à noter cependant des différences individuelles en ce qui concerne les espèces et même les genres pour des moutons placés sur le même régime. Ainsi, dans le régime $I$, les espèces du genre Diplodinium du mouton 70 sont différentes de celles des moutons 68 et 32 , en outre, il comprend le genre Epidinium nullement représenté ailleurs dans les rumens de moutons étudiés. Le mouton 70 a, en ce qui concerne ses grandes espèces, une population type B ainsi classée par EADIE, I967, pour les différencier (à cause de leur antagonisme) du type A, que l'on retrouve dans les moutons des régimes II et III et les autres moutons du régime I.

\section{Quantitativement.}

La population ciliaire des moutons soumis au régime foin de graminées et foin de graminées-tourteau de soja est de l'ordre de 15 à $22 \times 10^{4} / \mathrm{ml}$; les grandes espèces représentant une population de l'ordre de $30 \times \mathrm{IO}^{3} / \mathrm{m} 1$. De même, lors de nouveaux essais, nous avons observé chez les moutons au régime foin de graminées une population de grandes espèces composée de : Holotriches $15 \times 10^{3} / \mathrm{ml}$, Diplodinium II $\times \mathrm{IO}^{3} / \mathrm{ml}$; chez les moutons au régime foin de graminées-protéine de soja, cette population comprend : Holotriches $16,5 \times 10^{3} / \mathrm{ml}$, Diplodinium $\mathrm{I} 7 \times \mathrm{IO}^{3} / \mathrm{ml}$. Quant à la population ciliaire des moutons soumis au régime à base de concentrés (orge), elle est de l'ordre de $200 \times 10^{4} / \mathrm{ml}$, c'est-à-dire dix fois plus importantes, mais les grandes espèces sont insuffisamment représentées pour pouvoir être dénombrées. Cette population est essentiellement riche en Ciliés du genre Entodinium pour lesquels l'amidon est la source organique indispensable au maintien de leur culture in vitro (Coleman, I963; Hungate, I966). Plusieurs genres de Protozoaires participent à la fermentation de l'amidon dans le rumen : Entodinium, Epidinium, Isotricha. 
Ën ce qui concerne l'influence du $\mathrm{pH}$ sur la population faunale, il est connu que lorsque le $\mathrm{pH}$ descend en dessous de 5,0, la population décroît notablement jusqu'à disparaître, ce qui est le cas dans certains régimes à base de céréales. Dans nos essais avec le régime orge, le $\mathrm{pH}$ s'est maintenu entre 6 , I et 6,8 du fait de la présence dans le régime de paille et d'urée. Ces $\mathrm{pH}$ correspondent aux limites optimum permettant le développement des populations ciliaires. De sorte que nous n'avons pu observer de grandes variations dans les populations pour une chute de $\mathrm{pH}$ dans cette zone optimum. Ainsi le $\mathrm{pH}$ baisse après chaque repas et plus nettement dans le régime III au repas du soir (orge-paille), mais cette variation du $\mathrm{pH}$ est peu reflétée dans la population. EADIE et ses collaborateurs (I970) observent également, sur un régime comportant $50 \mathrm{p}$. Ioo de foin et $50 \mathrm{p}$. Ioo d'orge, un développement très important de la population microbienne du rumen et plus particulièrement des Protozoaires $\left(3,34 \times 10^{6} \mathrm{P} / \mathrm{m} 1\right)$. Cette microfaune est largement composée de petites espèces (75 p. 100) (Entodinium). Par contre, ces auteurs observent sur un régime à base d'orge donné ad libitum, la disparition complète de cette microfaune.

\section{Influence de l'azote ammoniacal.}

L'urée semble permettre le maintien et l'accroissement d'une population de petites espèces. Dans le présent travail, il apparaît que la concentration des Ciliés est plus élevée là où en moyenne le taux d'azote ammoniacal est le plus élevé.

Lors de récents essais, dans un régime à base de céréales (orge) où nous avons remplacé l'urée par une source azotée protéique (protéine de soja), nous avons constaté une population ciliaire globale inférieure à celle présente dans le régime orgeurée $\left(200\right.$ à $300 \times 10^{4} \mathrm{P} / \mathrm{ml}$ pour le régime orge-urée; $140 \times 10^{4} \mathrm{P} / \mathrm{ml}$ dans le régime orge-protéine de soja) mais les grandes espèces (Holotriches, Polyplastron) sont davantage représentées.

De même, PURSER et Morr (I 966 ) observent une concentration plus élevée de Protozoaires dans le rumen, lorsque l'urée est ajoutée à la ration (luzerne-avoine) comparativement au témoin et la concentration d'Entodinium est également plus élevée que dans le cas où la ration comporte du gluten.

In vitro nous avons précédemment observé (BonHomme, 1973) que l'addition d'urée à de faibles concentrations (O,OI p. IOO) aux glucides (amidon et cellulose) introduites dans les cultures de Ciliés du rumen, permet une augmentation de la durée de survie de ces Protozoaires de l'ordre de 30 p. roo environ. Or, l'urée est rapidement hydrolysée en ammoniaque dans le rumen. Les Bactéries élaborent leur protéine à partir de l'azote ammoniacal, cette protéine bactérienne est à son tour utilisée par les Ciliés ainsi que nous l'avons montré (BonHomme, 1968) pour élaborer leur propre protéine.

Pour de nombreux auteurs, dont PURSER et MOIR (I966), l'utilisation directe de l'azote ammoniacal et des simples composés azotés par les Protozoaires est impossible. D'autre part, ABE et KANDATSU (I968) ont observé que l'incorporation de ${ }^{15} \mathrm{~N}$ à partir de 1'ammoniaque est plus lente chez les Protozoaires que chez les Bactéries.

Il a été par ailleurs observé chez les Ruminants faunés, une concentration d'ammoniaque dans le rumen plus élevée que chez les Ruminants défaunés. D'après LUTHER et coll. (rg66), l'addition de Protozoaires aux fermentations bacté- 
riennes augmente la production d'ammoniaque de $48 \mathrm{p}$. Ioo et de $15,7 \mathrm{p}$. Ioo pour les rations respectivement pauvre et riche en concentrés (20 et $80 \mathrm{p}$. Ioo de concentrés).

Cette étude préliminaire montre que la nature chimique, la composition du régime influencent quantitativement et qualitativement la faune du rumen. Dans un régime composé uniquement de fourrage (foin de graminées), tous les genres et plus particulièrement les grandes espèces (Diplodinium) sont représentés. Il en est de même lorsque le tourteau de soja est associé à la ration. Les populations les plus nombreuses sont trouvées dans les régimes riches en céréale associée à de l'urée. Elles sont dix fois plus abondantes et sont surtout composées de petites espèces : le genre Entodinium est le plus représenté. Enfin lorsque le régime conduit à une concentration élevée d'ammoniaque dans le rumen, on observe également une concentration élevée de Ciliés. L’urée, ainsi, favoriserait le développement des petites espèces de la microfaune. Cette étude du rôle de quelques rations alimentaires sur la population faunale du rumen doit être poursuivie afin de complémenter nos résultats concernant en particulier le rôle de l'urée dans la physiologie de ces Ciliés.

Ręu pour publication en mars 1974 .

\section{SUMMARY}

QUALITATIVE AND QUANTITATIVE VARIATIONS

OF THE FAUNA IN SHEEP RUMEN DEPENDING ON THE TYPE OF DIET

During in vivo studies we observe qualitative and quantitative variations in the protozoa population, depending on the different rations offered twice a day. The diets have a barleyurea base which favorizes a very large development of ciliates, most of the genus Entodinium. Diets composed of meadow hay favorize a more varied genus population, the majority being large species. The number of protozoa is about ten times less with these diets. Measurements of $\mathrm{pH}$ and ammonia nitrogen content are carried out to study the relations between certain chemical conditions in the rumen and the development of the microfauna.

\section{RÉFÉRENCES BIBLIOGRAPHIQUES}

Abe M., Kandatsu M., I968. Untersuchungen zur Verwertung von nicht-Protein Stickstoff-Verbindungen beim Wiederkauer. Archiv. fur Tierernährung, 18, 247-263.

Bonhomme-Florentin A., I968. Sources azotées des Ciliés Oligotriches du genre Entodinium, Protistologica, 4, 507-5II.

Bonhomme-Florentin A., 1973. Contribution à l'étude de la physiologie des Ciliés Entodiniomorphes endocommensiux des Ruminants et des Équidés. Thèse (Reims) $\mathrm{n}^{\circ} 7923$.

Christiansen W. C., Woods W., Burroughs W., ig64. Ration characteristics influencing rumen Protozoa populations. J. Anim. Sci., 23, 984-988.

Coleman G. S., r963. The growth and metabolism of rumen Ciliate Protozoa. Symp. Soc. Gen. Microbiol., 13, 298-324.

EAdiE J. M., I967. Studies on the microbiology and biochemistry of the rumen in cattle given different quantities of a pelleted barley ration, Brit. J. Nutr., 24, I57-I77.

Eadie J. M., Hyldgaard-Jensen J., Mann S. O., Reid R. S., Whitelaw F. G., I97o. Observations on the microbiology and biochemistry of the rumen in cattle given different quantities of a pelleted barley ration. Brit. J. Nutr., 24, I57-177. 
Luther R. A., Trenkle A., Burroughs W., 1966. Influence of rumen Protozoa on volatile acid production and ration digestibility in lambs. J. Anim. Sci., 25, I I I6- I 22.

Naga M. A., Abou Akmada A. R., El Shazly K., 1969. Establishment of Rumen Ciliate Protozoa in cow and water Buffalo (Bos bubalus L.) calves under late and early weaning systems. J. Dairy Sci., 52, IIO-II2.

Purser D. B., Moir R. J., r966. Dietary effects upon concentrations of Protozoa in the rumen. $J$. Anim. Sci., 25, 668-674.

WARNer A. C. I., 1962. Some factors influencing the rumen microbial population. J. Gen. Microbiol., 28, $129-146$. 